\title{
Profesionales que emigran. Una comparación entre enfermeras e ingenieros mexicanos en Estados Unidos
}

\author{
Professionals who emigrate. A comparison between \\ Mexican nurses and engineers in the United States
}

doi: http://dx.doi.org/10.32870/

Alfredo Hualde Alfaro

espiral.v24i70.6119

Yetzi Rosales Martínez*•

\begin{abstract}
Resumen
Este trabajo explora los procesos migratorios y la inserción laboral de los ingenieros y enfermeras mexicanos en Estados Unidos. Se examina cómo los estándares de certificación, la capacidad de agencia de los migrantes y la intervención de diversos actores en la migración contribuyen a construir trayectorias laborales diferenciadas al interior de cada grupo profesional y entre ellos en el mercado de trabajo estadounidense. Otros factores estructurales que ayudaron a comprender los procesos estudiados fueron las motivaciones para migrar, las redes familiares y profesionales y el rol de género.
\end{abstract}

Palabras clave: migración calificada, regulación, transferencia de habilidades, profesionales, Estados Unidos.

\begin{abstract}
This paper explores the migration processes and labor incorporation of two professional groups of Mexicans in the United States: engineers and nurses. Certification standards, the ability of migrants to act as agents, and the involvement of various actors in the migratory process are analyzed in order to understand how differentiated labor paths are built within and between each occupational group in the US labor market. Other structural factors that helped to understand the processes studied were the motivations to migrate, family and professions networks, and gender.
\end{abstract}

Keywords: skilled migration, regulation, skills transfer, professionals, United States.

\footnotetext{
- Profesor-Investigador del Departamento de Estudios Sociales, El Colegio de la Frontera Norte (COLEF), México. ahualde@colef.mx

- Profesora-Investigadora del Departamento de Estudios de Población, CONACYT-El Colegio de la Frontera Norte (COLEF), México._romy@colef.mx

Fecha de recepción: 12 de septiembre de 2016. Fecha de aceptación: 26 de mayo de 2017.
} 


\section{Introducción}

La migración de profesionales de México a Estados Unidos ha sido considerada como un fenómeno secundario frente al gran flujo de migrantes con escolaridad no universitaria. A pesar de ello, como se hace notar en este artículo, la regulación de las profesiones fue uno de los temas incluidos en el Tratado de Libre Comercio (TLCAN), y desde principios del siglo XXI se registran iniciativas que tratan de recuperar para México esa mano de obra calificada.

En cualquier caso, este relativo desinterés fue bastante persistente, pues todavía a principios del siglo XXI se anotaba que esta problemática, debatida en Europa y en América Latina, en México había sido tratada de manera episódica y poco documentada (Didou, 2004). Recientemente, se advierte un nuevo impulso en la investigación, sobre todo en lo que se refiere al análisis de las condiciones de trabajo y la relación entre niveles de escolaridad y ocupación (Calva y Alarcón, 2015; Lozano y Gandini, 2012; Lozano, Gandini y Jardón, 2015).

Como se decía, esto contrasta con la situación a escala internacional, donde desde los años sesenta del siglo Xx la migración de profesionales o migración calificada ha captado la atención de los académicos y de los Gobiernos por el perjuicio que podría ocasionar en los países de origen la pérdida de grupos de personas con alto nivel de escolaridad (brain drain) (Carrión y Hualde, 2013; Brandi, 2006). Particularmente, la migración de profesionales de la salud ha llamado la atención por la conformación de health clusters que agudizan los desequilibrios en la capacidad de los sistemas de salud para hacer frente a las necesidades de sus poblaciones (Connell, 2008).

A nivel internacional, el debate continúa, pero ahora se dice que la migración de profesionales también implica efectos positivos para el país emisor (brain gain) (Stark y 
Fan, 2007). Esta idea se ilustra enfáticamente con el ejemplo de los ingenieros indios que estudiaron y trabajaron en los Estados Unidos y a su regreso crearon empresas de alta tecnología en la India gracias a las redes profesionales construidas en ambos países (Iredale, 2001).

En el análisis de esta problemática han surgido nuevos temas y aproximaciones metodológicas a partir de los años ochenta. En primer lugar, se observa un reconocimiento cada vez mayor de la heterogeneidad de la migración. En segundo lugar, se le da más importancia al análisis de las políticas de migración y a sus consecuencias. En tercer lugar, se advierte un interés renovado por la calidad del trabajo de los profesionales y de las formas en que adquieren o transforman sus competencias laborales. En cuarto lugar, se constata que la migración relacionada con el sector corporativo aumentó con la globalización económica y la expansión de las empresas trasnacionales (Koser y Salt, 1997). Y, finalmente, se otorga mayor atención al surgimiento de intermediarios que obtienen ganancias de la migración al proveer información y conectar a los trabajadores extranjeros con los empleadores en el contexto receptor (Hernández León, 2012).

Este trabajo compara dos grupos de profesionales que ilustran la heterogeneidad mencionada tanto en lo que se refiere a su composición por sexo como a los distintos estándares de certificación que deben cumplir para practicar sus conocimientos en el lugar de destino. Interesa documentar de qué manera se da el proceso de incorporación laboral de los profesionales al mercado de trabajo, enfatizando el aspecto regulatorio y la transferencia de habilidades, y describir tanto la capacidad de agencia de los propios migrantes como la de otros actores que intervienen favoreciendo o dificultando dicho proceso. Por capacidad de agencia se entiende la acción proactiva, los recursos personales y las estrategias encaminadas a lograr intencionalmente ciertos objetivos (Giddens, 2006). 
Se eligió el análisis comparativo cualitativo como estrategia metodológica para sistematizar trayectorias migratorias y laborales en un mismo país de recepción. La justificación de su uso en este estudio está en su potencial para profundizar en una muestra relativamente reducida de casos empíricos y encontrar explicaciones causales a partir del análisis de procesos sociales. Este método indaga las combinaciones causales (y no causas aisladas) que llevan a obtener resultados diferenciados en un contexto dado, así como a identificar las especificidades adherentes a cada caso. Estos fines se ajustan a la naturaleza compleja del problema de investigación aquí expuesto (Ariza y Gandini, 2012).

Las dimensiones de análisis que legitiman la homogeneidad de la comparación y brindan una identidad de clase (Zoya, 2011) a ambos grupos profesionales son la capacidad de agencia, la regulación migratoria y la inserción laboral.

En cada una de las profesiones seleccionadas, el tema es relevante por diferentes razones. En el caso de los ingenieros, se trata de una de las profesiones emblemáticas en las economías contemporáneas, desde el periodo de industrialización fordista hasta la actual economía del conocimiento. Para algunos autores, los ingenieros son "los creadores de los aspectos más importantes de nuestra civilización" (Lasserre, 1989, p. 9). Aunque la hipérbole citada sea discutible, no cabe duda que los ingenieros y profesionistas afines son percibidos como actores centrales de la economía del conocimiento.

El caso de la enfermería resalta por la importancia creciente del cuidado de la salud a nivel mundial debido entre otras cosas, al envejecimiento de las poblaciones. A pesar de ser una profesión históricamente subordinada dentro de la estructura hospitalaria, la enfermería ha sido altamente requerida desde la época de la Posguerra. Hoy en día, continúa siendo una profesión demandada en países desarrollados con transiciones demográficas avanzadas y 
un stock insuficiente de personal nativo en su sector salud. Estados Unidos es uno de los principales países receptores de personal de enfermería en el mundo (Acacio, 2011; Squires y Beltrán, 2013). La demanda de estos profesionales se ha reflejado en el diseño e instrumentación de políticas de admisión temporales y específicas para este personal, como la visa H1-A y H1-C (Joel y Kelly, 2002).

Vale la pena mencionar que este flujo migratorio ocurre en un contexto fuertemente asimétrico: entre México y Estados Unidos, los sueldos han sido históricamente muy desiguales, y aún en la actualidad se mantiene una brecha importante. Mientras que en México el salario mínimo mensual es de USD 170.00, en Estados Unidos este es de USD 1257.00 (OIT, 2010). La segunda característica de este contexto binacional es la fuerte integración económica y social entre los dos países.

En este artículo, se presentan resultados basados en entrevistas a profundidad realizadas a enfermas e ingenie$\operatorname{ros}^{1}$ mexicanos que residen en diversos lugares de Estados Unidos. ${ }^{2}$ Cada guía de entrevista está ligada a distintos proyectos de investigación con objetivos similares sobre cada grupo ocupacional. Se entrevistó a veintidós ingenieros y veintiocho enfermeras, contactados con ayuda de organizaciones profesionales e instituciones educativas en México y Estados Unidos. Prácticamente todos los entrevistados eran de nacionalidad mexicana, concluyeron sus estudios en México, ${ }^{3}$ tenían experiencia laboral en Estados Unidos y,

I. Se utiliza de manera genérica el término enfermeras por ser las mujeres una mayoría en esta profesión.

2. Las entrevistas y algunos resultados son parte del trabajo de investigación que dio lugar a dos tesis de doctorado. En el caso de los ingenieros, se trata de Carrión (20I4), mientras que la tesis sobre enfermeras es de Rosales (20I4).

3. En el caso de enfermería, se trabajó en la región San Diego-Los Ángeles y se incluyeron informantes con estudios a nivel general y licenciatura. En el caso de los ingenieros, todos excepto una ingeniera concluyeron sus estudios universitarios en México, pero una parte de ellos estudió posgrados en Estados Unidos. 
en el caso de las enfermeras, habían intentado revalidar sus estudios en ese país (con o sin éxito).

Para abordar el tema, en una primera sección se revisan los enfoques teóricos más significativos en las investigaciones sobre migración, destacando aquellos que se refieren a la migración de profesionales. En la segunda sección, se exponen datos cuantitativos que dan una idea de la magnitud del fenómeno y de las condiciones salariales de ingenieros y enfermeras en Estados Unidos. A continuación, se presentan los resultados de la investigación y, finalmente, en las conclusiones se reflexiona acerca de los resultados obtenidos.

\section{Migración y profesiones}

El concepto de profesión adquiere relevancia en el contexto de la migración calificada porque no sólo se controla la entrada de personas a cierto país, sino que también se regulan sus competencias. Una expectativa generalizada es que el migrante ejerza su profesión en la sociedad a la que llega. No obstante, la regulación de las profesiones a través de las fronteras puede convertirse en un obstáculo debido a las distintas normas de certificación entre país de origen y destino. Las trayectorias migratorias y laborales tanto de ingenieros como de enfermeras se rigen por elementos implícitos a su profesión que se vinculan con el grado de autonomía de la misma, los procesos de certificación y el concepto de cierre social.

En el siglo XXI, la caracterización flexible, cambiante y abierta de las carreras se distancia de visiones clásicas como la teoría dual del mercado de trabajo, formulada por Doeringer y Piore en los años setenta. Estos autores distinguían entre un sector primario, con buenas condiciones de trabajo y estabilidad, donde habitualmente se empleaban los trabajadores calificados, y un sector secundario, con peores 
salarios y condiciones de inestabilidad más evidentes. En la teoría de la segmentación triple, derivada de la anterior, se destaca además la presencia del segmento profesional (Sengenberger, 1988).

En ambos enfoques, sin embargo, la estabilidad rige las carreras de los trabajadores calificados, y el compromiso, la confianza y la lealtad con la empresa constituyen los fundamentos de una institucionalidad destinada a reproducir organizaciones y empleos basados en reglas, normativas y certificaciones que reducen la incertidumbre de empleados y empleadores. Sin embargo, en los estudios sobre carreras sin fronteras son frecuentes los trabajos que enfatizan los beneficios derivados de cambiar de empresa y de empleo. El aprendizaje, la satisfacción en el trabajo o el estatus son motivaciones importantes entre los trabajadores altamente calificados, además de los salarios (Benner, 2006).

Por otro lado, las teorías clásicas de las profesiones se basan en tres elementos importantes. En primer lugar, la idea de que la profesión permite un "cierre" (social closure) de los mercados de trabajo y asegura un monopolio a los profesionales en el ejercicio de la profesión. La segunda idea es que los profesionales se distinguen de otros trabajadores por su autonomía (Becker, 2009). En la medida en que poseen un saber experto, los profesionales gozan de una gran capacidad de decisión en su vida laboral. El tercer elemento es que las profesiones están reguladas por un entramado institucional regido por las normas e instituciones del Estado-nación (Hualde, 2000; Dubar y Tripier, 1998; Faulconbridge y Muzio, 2012). En esta perspectiva, las instancias que otorgan legitimidad a los saberes adquiridos a lo largo de una formación para una profesión son las instituciones educativas, las asociaciones profesionales y otras organizaciones similares.

Estos elementos constitutivos han sido criticados a la luz de la evolución de algunas profesiones. Por un lado, la idea 
del cierre del mercado de trabajo depende de la fortaleza de la profesión y de su capacidad para imponer un monopolio legítimo dentro de una jurisdicción específica (Abbott, 1988). Históricamente, los ingenieros no se han distinguido por esta capacidad. ${ }^{4}$ Por otro lado, esta autonomía fue erosionándose en la medida en que los profesionales se incorporaron a las organizaciones como asalariados (Demazière y Gadea, 2009, p. 21; Freidson, 2001; Hualde, 2000; Panaia, 2008).

Además, en el caso de la enfermería, es preciso mencionar que estos profesionales típicamente se incorporan a una estructura organizativa y de poder donde el control monopólico es ejercido por la profesión médica. De esta forma, la incipiente y parcial autonomía de la enfermería la ha colocado conceptualmente en el estatus de cuasiprofesión o profesión paramédica (Pacheco, 1997; Hugman, 1991).

El tercer elemento que ha sufrido modificaciones, como se verá a continuación, es el de la dependencia exclusiva de la profesión respecto de la jurisdicción del Estado-nación.

\section{La participación pública y privada en la regulación de las profesiones}

Se ha escrito que la regulación de las profesiones en ocasiones está a cargo del Estado (regulación desde arriba) y que en otras viene desde abajo, es decir, de las asociaciones, colegios e instancias similares que agrupan y representan a los profesionales. Sin embargo, es más frecuente que varios actores públicos y privados intervengan con distintos grados de influencia o de poder en función de entornos nacionales específicos y de la historia y las tradiciones de la profesión. 
En lo que se refiere a las ingenierías en México, el Estado, a través de la Secretaría de Educación Pública (SEP), otorga la cédula profesional y los títulos en las universidades públicas, pero también la Academia de Ingeniería de México tiene un papel destacado, y las universidades privadas, como el Tecnológico de Monterrey, son influyentes por la gran preferencia del sector empresarial hacia los ingenieros graduados de ellas y su énfasis en una formación internacional con dominio del idioma inglés.

En el caso de la enfermería, su estatus profesional ha sido influenciado por la acción perjudicial o benéfica de actores privados y sociedad civil organizada: por un lado, la proliferación de escuelas privadas que funcionan al margen de la SEP impartiendo enfermería a nivel técnico y profesional, sin los estándares mínimos de calidad y, por otro, la labor de asociaciones y colegios de enfermería que impulsan la profesionalización del gremio, privilegiando los estudios de nivel licenciatura y posgrado (Nigenda y Ruiz, 2010).

El papel del Estado como legitimador también se diluye relativamente por la migración creciente de profesionales hacia otros países. Los procesos de globalización, agudizados desde la segunda mitad del siglo Xx, han dado lugar a nuevas perspectivas en el análisis de la migración que ponen especial atención a la migración calificada. Se ha destacado que los acuerdos políticos y económicos, como la Unión Europea y el TLCAN, facilitan el flujo de trabajadores calificados. ${ }^{5}$ Sin embargo, estos procesos tampoco se dan de manera sencilla ni lineal. Para el caso del TLCAN, se han dado negociaciones donde participan los Gobiernos y las asociaciones profesionales de los tres países involucrados en él, con resultados limitados debido principalmente a

5. Una medida en sentido contrario es la decisión del Gobierno suizo, a principios de 2014 , de restringir la entrada a Suiza de trabajadores calificados europeos. 
las dificultades propias de homologar planes de estudio (Rodríguez Gómez, 2013).

Un segundo factor importante en la migración calificada es la internacionalización de la educación, que hace que los residentes de los países en desarrollo vean los estudios de grado occidentales como un salvoconducto (ticket) para el empleo en los países más industrializados. El reconocimiento de los que estudian en estos países no es un problema para los empleadores y esos profesionales pueden integrarse rápidamente en el mercado de trabajo (Iredale, 2001; Zhou y Hsu, 2011). Además, cada vez son más frecuentes los títulos reconocidos simultáneamente por varias universidades en distintos países. ${ }^{6}$

Por otro lado, en contraste con las visiones tradicionales acerca de las carreras profesionales, en nuevos tipos de análisis cobran importancia los siguientes aspectos: los saberes móviles, el conocimiento y habilidades que se adquieren en múltiples empresas, la identificación personal con un trabajo significativo, acciones de aprendizaje en el trabajo, el desarrollo de múltiples redes, las relaciones de aprendizaje entre pares y la responsabilidad individual en la administración o gestión de la propia carrera (Sullivan, 1999).

Esta visión, mucho más flexible y cambiante, no borra las diferencias entre profesiones. La diferencia más pronunciada se encuentra entre las profesiones ligadas al cuidado de la salud (médicos y enfermeras) y las relacionadas con las tecnologías de la información (TIC). En las primeras, los requisitos de certificación exigidos por las asociaciones profesionales y los Gobiernos siguen imponiendo fuertes barreras. En cambio, en las profesiones ligadas a las TIC,

6. Ese el caso de uno los ingenieros entrevistados, que poseía una maestría en administración de sistemas digitales reconocida por universidades de México, Holanda, España y Estados Unidos. 
la industria es muy fluida en lo que se refiere a los requerimientos de competencias; es internacional y hay poco impacto de los contextos culturales particulares; está dominada por el idioma inglés; la experiencia en el empleo es la forma más importante de adquirir capital y llegar a ser polivalente; se observa un alto nivel de movilidad interregional y entre países; hay potencial para el retorno de la inversión y de la migración; finalmente, las profesiones están poco reguladas por sindicatos u otros mecanismos (Iredale, 200I, p. 13). ${ }^{7}$

Dentro de las teorías de la migración, algunos analistas se han centrado en la inserción, ya sea considerando el segmento del mercado laboral al que se incorporan los migrantes o el tipo de ocupación que desempeñan. Aunque los contextos de recepción pueden ser muy variados, se han propuesto tres tipos ideales: desfavorable, neutral y favorable (Portes y Böröcz, 1989).

En el tipo de recepción favorable, los inmigrantes son recibidos de forma activa y legal por parte del Gobierno receptor. En el caso de los Estados Unidos, existe una legislación que crea condiciones para que los migrantes desarrollen sus habilidades en segmentos laborales profesionales que a veces requieren competencias complejas. Por ejemplo, los profesionales pueden ingresar a Estados Unidos con cuarenta y un tipos de visas considerando subclases en cada rubro. Sin embargo, las dos más importantes son la visa $\mathrm{H}-1 \mathrm{~B}$, para ocupaciones en los campos donde se requieren conocimientos altamente especializados, y la visa TN, especial para ciudadanos de Canadá, Estados Unidos y México, que tiene su origen en el TLCAN. En el 2011, el 59.6\% de las 106445 visas H-1B otorgadas por Estados Unidos fueron para ocupaciones relacionadas con la computación, ingeniería y arquitectura, lo cual lleva a suponer que, en comparación con migrantes de otras profesiones, los que

7. La cita es traducción personal del original en inglés. 
se dedican a estas últimas tienen más probabilidades de emplearse con mejores condiciones en el mercado laboral estadounidense.

Con respecto a la visa TN, se ha reportado un uso menor en una primera etapa pues hasta el año 2004 nunca se habían superado las 1000 visas otorgadas, cifra muy inferior a la cuota inicial de 5500 anuales. A partir del año 2005, se produjo un aumento importante, de manera que sólo en el año 2012 se otorgaron 7601 visas. Una tendencia similar se observa cuando se considera el total de visas para profesionales, que alcanzaba 26000 en el 2012. Como porcentaje del total de este tipo de visas, las TN fueron al principio un porcentaje insignificante, pero en el 2012 representaron ya el 28.9\% de las otorgadas a mexicanos (Rodríguez Gómez, 2013, pp. 205 y ss.).

Las perspectivas teóricas descritas permiten entender las hipótesis relativas a las ventajas que pueden obtener los profesionales que deciden emigrar y las barreras que limitan su incorporación en los mercados laborales. Por un lado, el interés de los países desarrollados se traduce en facilidades para atraer mano de obra calificada, que no decrece ni siquiera en tiempos de crisis (Lozano, Gandini y Jardón, 2015). Sin embargo, tanto la movilidad como las condiciones de empleo están condicionadas por características individuales de los migrantes, por sus estrategias y por elementos contextuales que cambian según coyunturas políticas o económicas.

El tercer factor a tomar en cuenta es la propia profesión, que en el caso de los ingenieros, especialmente en las ingenierías más recientes, no está regulada por certificaciones exigentes para acceder al mercado de trabajo, lo que sí sucede en el caso de las profesiones médicas.

Finalmente, es preciso tomar en cuenta factores relativos a la relevancia explicativa que tiene la composición por sexo en las profesiones. En la siguiente sección se resumen 
estimaciones cuantitativas de la migración de ingenieros y enfermeras mexicanas.

\section{La migración de profesionales mexicanos hacia} Estados Unidos

Algunos autores estiman que en el año 2012 casi 600000 migrantes mexicanos con licenciatura residían en los Estados Unidos (Lozano y Gandini, 2012; Lozano, Gandini y Jardón, 2015). Según los mismos autores, este tipo de migración creció un 35\% entre el año 2006 y el año 2012 (Lozano, Gandini y Jardón, 2015, p. 37), y de acuerdo con Calva y Alarcón (2015), el crecimiento fue de 85\% entre 2000 y 2011. Otros estudios calculan en 357000 el número total de profesionistas mexicanos que migraron a países de la OCDE, aproximadamente 16\% del total, siendo México el país que ha registrado la mayor tasa de crecimiento de este tipo de migración (270\%) entre los países de América Latina y el Caribe entre 1990 y 2007 (Ros, 2013).

\section{I. El caso de los ingenieros migrantes}

Se puede estimar que el número de ingenieros mexicanos en Estados Unidos asciende a 91 000. Ahora bien, dado que un tercio realizó sus estudios universitarios en los Estados Unidos, la cifra más probable es de 60 000. Si se toma el ingreso como un indicador de la situación laboral de los ingenieros, se observa que los que estudiaron en México ganan en promedio USD 45000.00 , en tanto que los que estudiaron en Estados Unidos perciben USD 53 000.00. Esta diferencia está positivamente relacionada con el dominio del inglés, sobre todo entre los que tienen un muy buen nivel del idioma (Carrión, 2014).

El número de ingenieros mexicanos que nacieron y estudiaron en México y ahora residen en Estados Unidos, alrededor de 60 000, supone un 4\% de la cifra de perso- 
nas que tienen estudios afines a la ingeniería en México. De los migrantes mexicanos con educación superior, los ingenieros y tecnólogos representan el segundo grupo en importancia, casi el 20\% del total, tan sólo por detrás de los egresados de escuelas de negocios. Sin embargo, también se ha destacado que tan sólo un tercio de los migrantes mexicanos con escolaridad universitaria estaban empleados en ocupaciones calificadas, el $20 \%$ se empleaban en ocupaciones técnicas, y el 47.6\%, casi la mitad, trabajaban en ocupaciones poco calificadas (Calva y Alarcón, 2015, p. 28). El mayor porcentaje de los empleados en ocupaciones calificadas se agrupaba en el área de docencia, capacitación y bibliotecas, en tanto que los ocupados en arquitectura e ingeniería representaban algo menos de un $10 \%$, porcentaje ligeramente más alto que los ocupados en el área de salud (Calva y Alarcón, 2015, p. 29).

El gremio de ingenieros fue uno de los pocos que a raíz de la firma del TLCAN participó y avanzó relativamente en conversaciones encaminadas a compatibilizar los requerimientos del ejercicio profesional respectivo. Esto es, durante varios años, representantes de varias profesiones se reunieron en los Comités Mexicanos para la Práctica Internacional con homólogos de Canadá y Estados Unidos, con resultados desiguales.

De acuerdo con Rodríguez Gómez (2013, p. 214), entre las profesiones que encontraron mayores dificultades para avanzar estuvieron las de salud (entre ellas enfermería), en tanto que las ingenierías se encontraban en una etapa intermedia en la acreditación y certificación de titulaciones. En el caso de los ingenieros, se firmó en 1995 en Washington un documento de recomendaciones sobre el reconocimiento mutuo de registro y licencias de ingenieros para las jurisdicciones de Canadá, Estados Unidos y México para la facilitación de su movilidad en el marco del TLCAN, pero no se logró un acuerdo de reconocimiento mutuo ni se puso 
en marcha un plan piloto, fase a la que sólo consiguieron avanzar los arquitectos (Rodríguez Gómez, 2013, p. 219).

\section{2. El caso de las enfermeras migrantes}

La migración del personal de enfermería mexicano hacia Estados Unidos ha sido escasamente investigada. A finales de la década de 1990, los registros sobre este éxodo comenzaron a ser difundidos principalmente a través de medios de comunicación. En la academia, las esporádicas publicaciones arrojaban algunas cifras desagregadas sobre la salida de enfermeras de algunas entidades mexicanas hacia Texas, Florida, California y Nueva York, lugares que presentaban déficit de estos profesionales (Arroyo de Cordero y Jiménez Sánchez, 2005; Nigenda y González Robledo, 2005). En términos regulatorios, se han evidenciado problemas en la transferencia de habilidades que dificultan la práctica de la enfermería de estos inmigrantes en Estados Unidos, orillándolos a subemplearse en otras ocupaciones (Rodríguez, 2004).

Durante el trienio 2009-2011, se registraron poco más de 17000 mexicanos con estudios de enfermería en Estados Unidos, de los cuales alrededor de la mitad terminó sus estudios en México (9 259). ${ }^{8}$ Este último grupo tiene una alta proporción de mujeres (93\%), con un promedio de edad de cuarenta y seis años. Un porcentaje elevado del grupo reportó no tener ciudadanía (72.1\%), lo que denota dificultades para insertarse legalmente en el mercado laboral. Esta situación se acentúa cuando el manejo del inglés del migrante no es fluido, situación en la que se halla casi la mitad de esta población (45.3\%). El ingreso promedio anual reportado por estos inmigrantes mexicanos fue de casi USD 14 000.00, cifra muy lejana al ingreso tabulado por el U. S.

8. Indicador generado a partir de la variable proxy "edad de entrada a Estados Unidos" a partir de los 22 años, según datos de American Community Survey, 2009-20II. 
Bureau of Labor en 2012 para una enfermera registrada, que era de USD 65000.00 anuales (Bureau of Labor Statistics, 2012).

Cuadro 1. Stock e ingreso promedio de las enfermeras e ingenieros mexicanos en Estados Unidos

\begin{tabular}{lcc} 
Características & Ingenieros & Enfermeras \\
Número (stock) $^{*}$ & 60000 & 9000 \\
Ingreso promedio anual (USD) $^{* *}$ & 45000.00 & 14000.00 \\
\hline
\end{tabular}

* Personas con estudios de licenciatura y posgrado.

** Para la conversión se calculó un tipo de cambio de MXN 18.00 por dólar.

Fuentes: Carrión (2014) y Ruggles, et al. (2015).

\section{3. Características de los entrevistados}

En el caso de los ingenieros, fueron entrevistados veinte hombres y dos mujeres que viven y trabajan en Estados Unidos. La mitad de los ingenieros tenía menos de cuarenta años en el momento de la entrevista (uno de ellos menos de treinta), siete entre cuarenta y cincuenta años de edad y cuatro eran mayores de cincuenta. La mitad de los ingenieros provenían de universidades públicas y la otra mitad de universidades privadas, a pesar de que en México el peso numérico de las universidades públicas en ingeniería es mayor que el de las privadas. ${ }^{9}$ Otro dato importante de esta muestra es que la mitad de sus integrantes habían cursado algún posgrado, lo cual introduce un sesgo hacia aquellos con mayores niveles educativos.

Parece importante resaltar tres rasgos que otorgaban una cierta ventaja competitiva a los ingenieros entrevistados. Por un lado, había una proporción relativamente alta de ellos que tenían padres con títulos de licenciatura y de posgrado; en segundo lugar, un número también importante de 
ellos procedía de universidades públicas o privadas de alto prestigio en el terreno de las ingenierías. Además, en dos casos, la carrera la habían cursado en universidades de California, en San Diego. La tercera ventaja potencial derivaba de que la condición para ser entrevistado era haber tenido una experiencia laboral como ingeniero en México, lo cual excluía a quienes emigraron sin haber ejercido previamente su profesión.

Por otro lado, las veintiocho enfermeras mexicanas entrevistadas tenían entre veinticinco y sesenta y un años de edad, con una media de treinta y nueve años. Procedentes de doce entidades federativas, Jalisco, Ciudad de México y Baja California ocupaban los tres primeros sitios en cuanto a lugares de origen. Todas habían egresado de escuelas públicas y cursado enfermería a nivel general (equivalente a cuatro años) y licenciatura (cinco años), incluido el servicio social. Cabe mencionar que la carrera de enfermería a nivel universitario fue creada en la década de los sesenta, pero tuvo mayor demanda como opción educativa desde principios de los noventa, cuando comenzó a impulsarse la profesionalización de la enfermería en México. El grupo de enfermeras llegó a California con distintos estatus migratorios y por motivaciones disímiles: algunas informantes contaban con redes familiares que les facilitaron el acceso a un permiso de residencia en Estados Unidos, y otras habían cruzado la frontera con visa de turista o TN. Al momento de la entrevista, nueve de ellas residían en California de manera irregular tras la expiración de su visa de turista. La mayoría migró para buscar la reunificación familiar y sólo once manifestaron haber migrado por razones laborales. Los años de entrada a Estados Unidos fluctuaron en el rango de 1985 a 2013. Respecto a su conocimiento del inglés, sólo las enfermeras que habían migrado mediante un permiso de trabajo consideraron su nivel de inglés como bueno, mientras que el resto lo calificó como malo o regular. 
4. La migración de los ingenieros y el papel de los actores en la regulación de las carreras

Una primera consideración a tomar en cuenta en la migración de ingenieros a Estados Unidos es que las motivaciones para trasladarse a dicho país son diversas, pero no derivan mayoritariamente de una necesidad económica perentoria, como ocurre habitualmente con los migrantes no calificados: el aprendizaje de nuevas técnicas y nuevas operaciones, y la inserción en sectores o actividades con mayor desarrollo tecnológico impulsan a muchos de ellos a cambiar de país.

Por otro lado, las formas de regulación de su profesión y la relación entre los diferentes actores que intervienen se relacionan con el tipo de trayectoria que construyen los profesionales a lo largo del tiempo. En dicha trayectoria resulta decisivo el papel de la empresa o empresas en las que se emplean, que para los entrevistados de este estudio fueron generalmente compañías trasnacionales. A continuación, se examina la forma en que se regula la ingeniería según los distintos tipos de trayectoria encontrados.

\section{I.Tipo I: trayectoria de empresa}

El tipo ideal de esta trayectoria es el de un ingeniero en sistemas computacionales de cincuenta años, egresado del Tecnológico de Monterrey, la institución de mayor prestigio en México entre los empleadores del sector privado. La trayectoria se tipifica sobre la base de su continuidad en una sola empresa que proporciona al ingeniero seguridad legal y laboral.

Este ingeniero, que también estudió una maestría en inteligencia artificial, desarrolló veinticinco años de carrera en una gran empresa trasnacional que lo llevó a vivir a México, Venezuela y Estados Unidos. En realidad, fue el único de los ingenieros entrevistados en el que se 
dio una estabilidad tan duradera en una sola empresa, lo cual lo asimila a lo que White (1958) denominaba hombre organización. Dos aspectos son importantes en este caso: su primer destino en el extranjero no fue Estados Unidos, sino Venezuela, por lo que su trabajo tenía una proyección latinoamericana al ser un departamento de ventas para toda la región. A Estados Unidos llegó como consecuencia de una decisión corporativa que convino a sus intereses.

Lo importante es que, a partir de un título de prestigio en México, la empresa se encargó de facilitarle los papeles necesarios para que su situación fuera legal y estable. En este sentido, para la empresa trasnacional, el hecho de tomar un papel protagonista en la regulación de la profesión la lleva a adoptar un papel de empresa protectora, que no es, como veremos a continuación, el mismo que desempeñan otras organizaciones. Este caso ilustra la gran importancia de la empresa como actor regulador que se relaciona, como dice otro ingeniero, con el aspecto legal del ámbito laboral:

[...] regularmente, cuando eres de México te vienes [a trabajar a Estados Unidos] con una visa de trabajo. Si tienes visa de trabajo, una de las condiciones es que no te puedes mover de compañía, porque te están haciendo lo que se llama spots, [lo que significa que] haz de cuenta [sic] que te está auspiciando la compañía; entonces si tú decides que quieres cambiar de compañía, tienes que volver a meter tus papeles de visa y te lo[s] pueden rechazar, entonces corres un riesgo muy grande. Ahora sí que te quedas como el perro de las dos tortas [sic] por andar queriéndote mover, pues al rato te quedas sin trabajo. Entonces, la única forma de salirte de trabajar y de cambiarte de compañía sería agarrando la residencia, [a la] que se le llama[n] la green card (entrevista personal, 2012). 


\section{2. Tipo 2: trayectoria de adaptación y riesgo:} outsourcing y certificaciones especializadas

La segunda trayectoria ideal típica es la de un ingeniero aeronáutico de cincuenta y cinco años, nacido en Tijuana y egresado del Instituto Politécnico Nacional, institución pública de gran prestigio en el ramo de las ingenierías en México. La racionalidad que subyace a esta tipología es la de quien siempre se mantiene en el sector de su especialidad, la aeronáutica en este caso, pero sin gozar del desarrollo de una política de protección, sino de mínimas garantías en el puesto de trabajo, lo que sucede en la mayor parte de las empresas.

El entrevistado acumulaba una cantidad importante de experiencias laborales en el sector y una trayectoria que lo había llevado desde la frontera de México con Estados Unidos hasta Montreal, Canadá, donde había trabajado para la empresa Bombardier, y luego de regreso a la región de San Diego, California.

A efectos del análisis de la regulación, vale la pena resaltar dos aspectos: por un lado, la persistencia de situaciones legales precarias, contratos en outsourcing y cambios de empleo por recortes o despidos; ${ }^{10}$ por otro lado, que en la trayectoria no hay barreras de certificación a la entrada, pero sí se percibe la exigencia de certificaciones permanentes propias del sector aeronáutico, para el caso del entrevistado mencionado, o exigencias comunes a las grandes organizaciones. Esta situación se asocia a empresas donde se han desarrollado estrategias flexibles de gestión de la mano de obra.

En cuanto al estatus legal, la residencia y el permiso de trabajo son inciertos. Los migrantes mayoritariamente

10. Tras varias rupturas laborales, el ingeniero entrevistado obtuvo en Canadá, por intermedio de la empresa Bombardier, un estatus legal que le permitió el regreso a los Estados Unidos y la obtención de la ciudadanía estadounidense desde su carácter de ciudadano canadiense. 
realizan el trámite por cuenta propia con la carta aval de la empresa, renuevan continuamente los permisos y básicamente se apegan a la visa del TLCAN, que es la más fácil de tramitar.

Finalmente, en esta trayectoria se valoran principalmente las competencias técnicas en sectores complejos, como el aeronáutico, donde dichas competencias son variadas y pueden cambiar con cierta rapidez según la actividad de la empresa.

\section{3. Tipo 3: trayectorias estratégicas}

La trayectoria ideal que se ha denominado estratégica la ejemplifica un ingeniero de cuarenta y cuatro años que nació en la Ciudad de México y estudió en la Universidad Autónoma Metropolitana, de dicha ciudad. En el momento de la entrevista estaba soltero y sin hijos. Después de trabajar en IBM en Guadalajara, México, estudió la maestría y el doctorado en arquitectura computacional en Indiana. La característica básica que da solidez y certidumbre a la trayectoria son los títulos de posgrado en Estados Unidos.

$\mathrm{Su}$ trayectoria la había desarrollado en varias empresas grandes hasta que tuvo la oportunidad de trabajar en San Diego, California, donde residía en el momento de la entrevista. Se denominó estratégica a esta trayectoria porque se advierte en el entrevistado un plan surgido de su experiencia laboral en IBM y de su conciencia acerca de la necesidad de aprender de manera continua y obtener títulos educativos.

A partir de este periodo de estudio e inserción posterior, los ingenieros con este tipo de trayectoria construyen redes sociales y empresariales que se convierten en un instrumento determinante en la búsqueda de empleo. Su capacidad de agencia se deriva del reconocimiento y estatus que les dan sus estudios en los Estados Unidos. Pueden elegir en buena medida la temporalidad y espacialidad de los 
cambios en su trayectoria. Su estatus legal inicia con una visa de estudiante y luego tramitan la residencia legal. En este caso, el actor legitimador es el sistema educativo del país de destino.

Un subtipo de la trayectoria estratégica puede denominarse trayectoria estratégica de aprendizaje, pero también puede considerarse como una trayectoria estratégica pragmática.

La subtrayectoria típica la encarna el ingeniero más joven de la muestra, un joven de veintinueve años que estudió ingeniería en computación en una universidad privada de Guadalajara. En México tuvo tres experiencias laborales en grandes empresas: IBM, Softtek e Hildebrando, las dos últimas las empresas mexicanas más grandes de software. De la primera salió a consecuencia de un recorte de personal y de las otras dos voluntariamente por aspectos del trabajo que no le gustaban y en búsqueda de superación profesional. El trabajo en Estados Unidos lo encontró a través de una reclutadora de una página web de una gran empresa que lo subcontrató. Para el momento de la entrevista, tenía un año en esta empresa, se sentía "a gusto", pero se manifestaba abierto a otras posibilidades, incluso a regresar a México.

Es necesario destacar que a lo largo de su breve vida profesional el entrevistado siempre tuvo trabajo, en todos los empleos asimiló y emprendió nuevos aprendizajes, y que contaba con un objetivo a largo plazo (ser arquitecto de software). No utilizaba redes sociales, puesto que él mismo se confesaba como alguien poco social, por lo que los cambios de empleo se habían dado en su caso a través de plataformas tecnológicas que ofrecen empleo.

En sus decisiones siempre había tenido presente su relación familiar (esposa e hijo), que había influido, por ejemplo, en la decisión de emigrar pues la esposa concebía este proceso como una aventura. Su relación con las empre- 
sas era pragmática, fuera de todo compromiso, y no estaba cerrado a regresar a México. En este caso, hay dos elementos a destacar: la experiencia previa en empresas de prestigio y el uso de plataformas electrónicas que, al parecer, tienen la confiabilidad suficiente como para proporcionar empleo a este tipo de profesionales.

\section{4. Tipo 4: trayectoria precaria}

La trayectoria ideal del tipo precaria es la de un ingeniero de treinta y cuatro años, originario de un estado del norte de México y egresado de ingeniería en sistemas computacionales de un instituto tecnológico público. La dificultad de encontrar trabajo en su ciudad de origen, a pesar de contar con alguna experiencia laboral, lo llevó a emigrar a la ciudad fronteriza de Tijuana. Tuvo empleos relacionados con el desarrollo de páginas web y supervisión de trabajos informáticos, hasta que decidió cruzar la frontera porque, dijo, no le pagaban como egresado. La contratación por medio de una agencia subcontratista de Tijuana, y el desarrollo posterior del trabajo, reflejan una acumulación de situaciones confusas y promesas falsas para conseguir los papeles, lo cual se suma a las propias limitaciones del sujeto, quien no hablaba inglés. Los arreglos entre las empresas mexicanas y las empresas subcontratantes de los Estados Unidos sitúan a los ingenieros contratados en situaciones muy inestables e inciertas.

Este tipo de trayectoria identifica a ingenieros que residen mayormente en ciudades fronterizas donde operan organizaciones que se dedican a la colocación de ingenieros en empresas de los Estados Unidos generalmente pequeñas y de bajo prestigio. Los ingenieros más proclives a recurrir a este tipo de reclutadoras son los que laboran en empleos caracterizados por la inestabilidad y los bajos salarios en México, y que ven una oportunidad de desarrollar su profesión en los Estados Unidos. En el caso de los que viven en 
Tijuana, la cercanía con Estados Unidos les permite cruzar a trabajar diariamente, y ocasionalmente a los que viven en otras ciudades fronterizas no tan cercanas. Principalmente cruzan con visa de turistas, y a veces como indocumentados. Es importante subrayar que la trayectoria de estos ingenieros se encuentra en una etapa relativamente temprana de su vida profesional.

\section{La migración de enfermeras.}

\section{Competencias explícitas y tácitas}

Las trayectorias del grupo de enfermeras entrevistadas estuvieron ligadas a la causa de su migración y al contacto con actores intermediarios antes y después de migrar. En este caso, es posible hablar de al menos dos trayectorias: la secuencial, con elementos inicialmente precarios, y la tardía, que es aún más precaria que la primera porque contiene experiencias de migrantes calificadas que al salir de México no transfirieron sus credenciales educativas y, por tanto, enfrentaron mayores retos laborales. Esta segunda trayectoria condensa historias migratorias asociadas a cuestiones familiares que, posteriormente, se empalman con la búsqueda de empleo y procesos de revalidación de estudios, una vez que las profesionales viven en Estados Unidos.

Las causas de la migración fueron de naturaleza laboral y familiar. Generalmente, la migración laboral tuvo como base la expectativa de obtener un mayor salario y desarrollo profesional, es decir, ocurrió a pesar de que tuvieran un empleo en enfermería en México. La decisión de migrar estuvo basada en el desempleo sólo en un caso. Por su parte, otro grupo de enfermeras buscó reunirse con otro migrante, su pareja o un familiar enfermo residente en aquel país, a 
quien le brindarían cuidados. ${ }^{11}$ Esta migración vinculada a lazos consanguíneos o conyugales encuentra sentido en el cumplimiento de roles tradicionales de género asignados a la mujer en sociedades como la mexicana y en la histórica composición feminizada de la enfermería.

Otra categoría analítica determinante en la migración e inserción laboral del personal de salud es la regulación profesional en la sociedad de destino. La práctica de la enfermería es ilegal en Estados Unidos sin la posesión de una licencia vigente expedida por los consejos estatales de enfermería. En este caso, en contraste con lo descrito para los ingenieros, la práctica de la enfermería está condicionada por una barrera a la entrada de tipo credencialista.

\section{I.Tipo I: trayectoria secuencial.}

De la precariedad al despliegue

Esta trayectoria queda ejemplificada en historias como la de Susana, enfermera licenciada de treinta y siete años, egresada de la Universidad de Guanajuato. Al momento de la entrevista, se encontraba en San Diego, California, divorciada y sin dependientes económicos. Su experiencia laboral en dos hospitales privados la había conducido a buscar mejores oportunidades en el extranjero al no ver satisfechas sus aspiraciones profesionales en México: "siempre sentí que no estaba practicando todos los conocimientos que aprendí en la universidad y me sentía frustrada profesionalmente, porque el sistema de salud en México limita mucho a las enfermeras" (entrevista personal, 2013a). Susana emigró mediante canales legales usando la visa TN, lo que hubiera sido poco probable sin la intermediación de una empresa

I I. En este último caso, aunque la migración respondió a un evento circunstancial, las experiencias recopiladas superaron, por mucho, el tiempo de estancia inicialmente planeado en EE. UU. debido al agravamiento del familiar enfermo o al establecimiento de lazos emocionales con otro inmigrante. 
subcontratista estadounidense que se había instalado temporalmente en la Ciudad de México en 2005.

Este tipo de trayectoria resulta de la combinación de expectativas individuales y factores coyunturales. Por ejemplo, la trayectoria secuencial en una profesión altamente regulada como la enfermería impone fuertes barreras a la certificación de conocimientos. Susana se preparó arduamente en México para mejorar su nivel de inglés y presentar el examen del Consejo Nacional de Enfermeras Registradas de Estados Unidos. En el proceso, la empresa subcontratista cerró sus puertas tras un desfalco financiero, y entonces Susana fue contactada por otra empresa del mismo giro, que se encargó de traducir sus certificados escolares, someter a revisión el plan de estudios de enfermería ante la instancia reguladora correspondiente en California y financiarle otro curso intensivo de inglés. Todo el proceso de revalidación de estudios y el trámite de licencia duró aproximadamente dos años.

La secuencia precaria descrita hasta aquí tuvo una temporalidad finita que fue definida por la duración del contrato con el intermediario. Después de dos años de trabajar en un hospital de prestigio en San Diego, finalizó el contrato que obligaba a Susana a compartir parte de su sueldo con el subcontratista. Su desempeño le valió ser contratada directamente por el hospital. En este punto, la trayectoria cambió radicalmente (turning point) para pasar a una secuencia de despliegue, caracterizada por una relativa estabilidad laboral, un salario competitivo y prestaciones, afiliación sindical y capacidad de agencia, esta última tras familiarizarse con el funcionamiento del hospital estadounidense, adquirir más habilidades técnicas y poder cruzar la frontera con su visa de trabajo: "Ahorita es diferente porque soy parte del staff del hospital, entonces ahora cuando me quedo más horas me pagan tiempo extra y [me dan] todos mis beneficios" (entrevista personal, 2013a).

\section{6}


Las enfermeras insertas en esta trayectoria procedían de las regiones centro-norte y centro-sur de México, por lo que sus desplazamientos no fueron transfronterizos. Asimismo, la transferencia de habilidades a través de exámenes de conocimientos técnicos y manejo del inglés fue un elemento decisivo, tanto en la interacción con los actores intermediarios como en su inserción laboral dentro del campo de la enfermería.

\section{2. Tipo 2: trayectoria tardía.}

\section{Reunificación familiar y trabajo}

Un caso típico de esta trayectoria lo ilustra Lucía, enfermera general egresada de la Universidad de Guadalajara, Jalisco. Después de haber ejercido la enfermería en México durante diez años en una institución pública de salud, renunció para emigrar junto con sus dos hijos a Los Ángeles, California, donde se encontraba trabajando su esposo. Esta trayectoria está fundamentada en el establecimiento de redes familiares de mexicanos en Estados Unidos. En este caso, las aspiraciones laborales no figuraron en la causa de la migración, por lo que la visa TN no se vislumbró como opción en ninguna de estas historias. Estas informantes arribaron a California como turistas o sin ningún tipo de permiso migratorio, por lo que sus competencias y experiencia profesional no se hicieron explícitas al momento del cruce. Este estatus migratorio y el escaso o nulo manejo del inglés coartaron la capacidad de agencia de estas enfermeras, tanto para cruzar libremente la frontera como para insertarse en el mercado laboral de la salud.

Esta trayectoria es relevante porque se registraron casos de movilidad laboral ascendente acompañados de una estancia prolongada en California, a pesar de existir numerosos obstáculos para ello. La inserción laboral fue tardía y comenzó en ocupaciones no relacionadas con la enfermería, pero con el tiempo la acumulación de informa- 
ción proporcionada por redes de familiares y organizaciones intermediarias y la regularización de su situación migratoria condujeron a las entrevistadas a practicar la enfermería en categorías laborales que implicaban distintas condiciones de empleo. Lucía, por ejemplo, comenzó tomando cursos de inglés y se certificó como asistente en enfermería. Esta licencia la autorizaba para brindar sus servicios en casas particulares a través de compañías subcontratistas, cuidando ancianos o enfermos terminales. Este tipo de inserción fue un nicho laboral común en esta trayectoria porque no se requería un estatus migratorio legal para su ejecución.

Por el contrario, la valoración de competencias en las categorías de enfermería de mayor prestigio y mejor pagadas sí requiere en California de un permiso de trabajo o la residencia permanente. ${ }^{12}$ Lucía logró obtener un permiso de residencia y, tras aprobar un examen de conocimientos, consiguió su licencia de enfermera vocacional licenciada (LVN, por sus siglas en inglés) para laborar en un hospital. Asimismo, la inserción laboral en algunos casos se retrasó o interrumpió por la incursión en la maternidad: "A mí me afectó tener mi bebé. Justo cuando el board me dio la carta de registro para el examen, ya no me inscribí porque el niño consumía mi tiempo. Es un obstáculo que me ha detenido para seguir con el trámite" (entrevista personal, 2013b). Cabe mencionar que esta trayectoria es similar a la experimentada por enfermeras que migraron por motivos laborales pero sin la intervención de subcontratistas en México y sin conocimiento sobre la necesaria transferencia de sus habilidades al país destino para practicar la enfermería.

12. Esta regulación no la aplican otros consejos de enfermería estatales, como el de Texas.

\section{8}



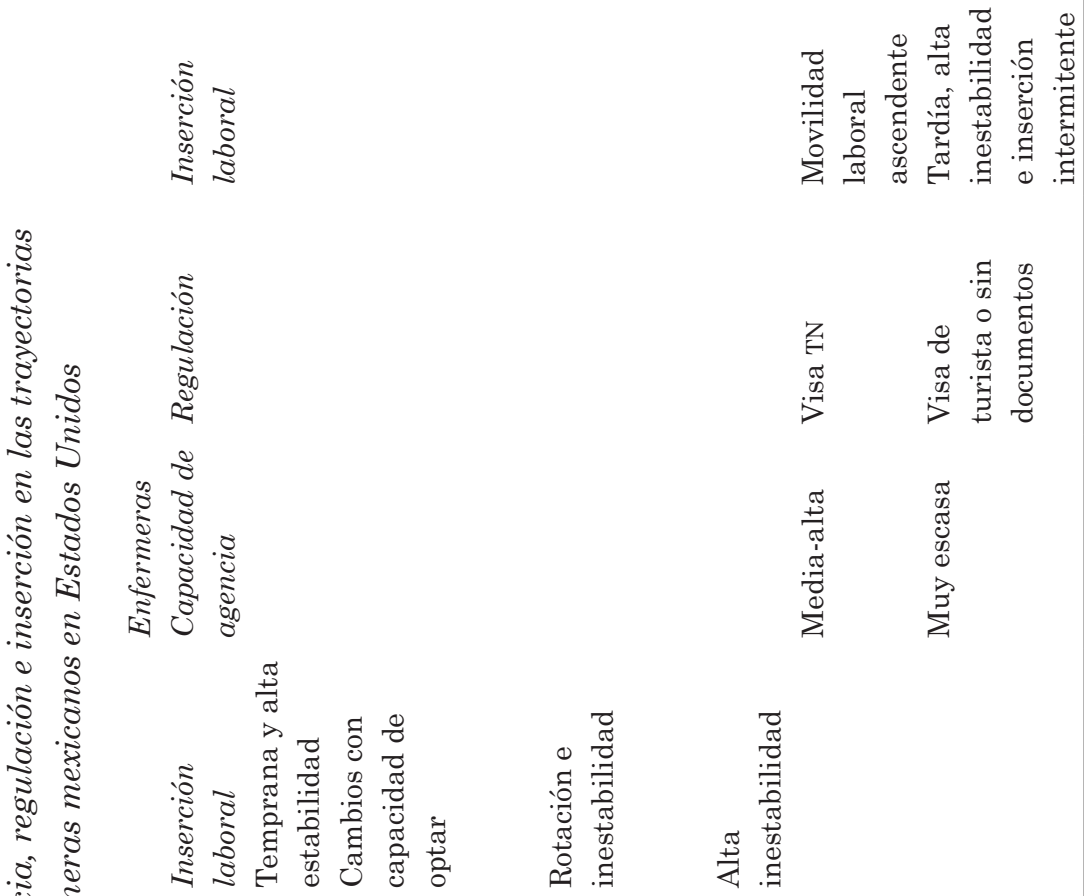

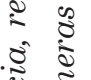
㤩总

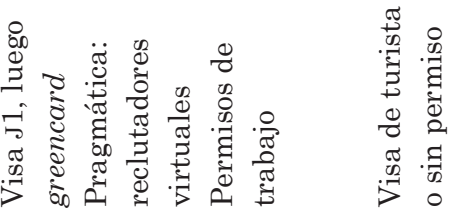

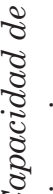

ค่
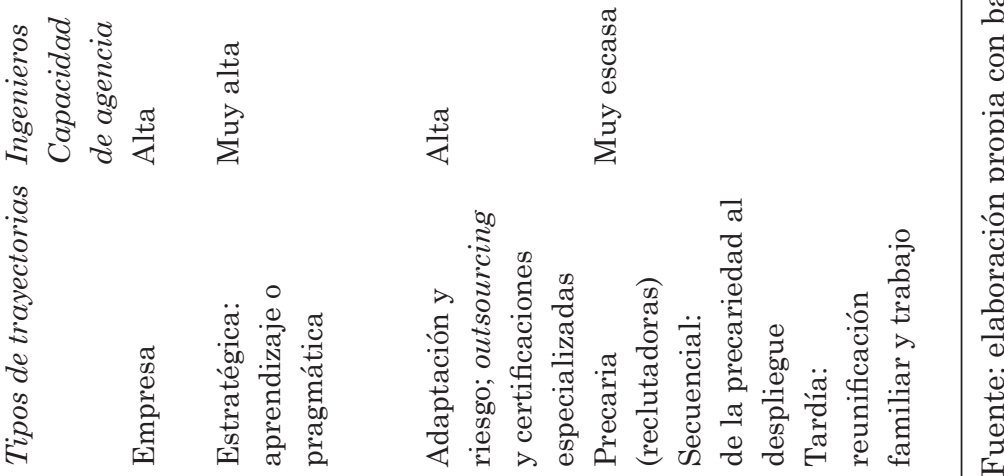


\section{Conclusiones}

La tipología propuesta ha permitido comparar similitudes y diferencias entre las trayectorias de enfermas e ingenieros mexicanos migrantes en Estados Unidos. Para cada profesión, se identificaron trayectorias heterogéneas configuradas en buena medida por los distintos estándares regulatorios que rigen a cada una, pero también se observaron, al interior de ellas, experiencias donde los recursos y la capacidad de agencia de cada grupo de individuos llevó a sus integrantes por rutas migratorias singulares e inserciones laborales distintas en el país al que emigraron.

Dos tipos de factores que podrían denominarse estructurales condicionan las diferencias en la trayectoria migratoria/laboral: por un lado, las barreras a la entrada, fuertes y de tipo credencialista, en el caso de las enfermeras, y más flexibles en el caso de los ingenieros, excepto en el caso de los civiles, que sí necesitan revalidar su título profesional en Estados Unidos. Por otro lado, la condición de género que mayoritariamente caracteriza a los integrantes de cada una de estas profesiones: al ser las ingenierías mayoritariamente masculinas, las trayectorias de los ingenieros se explican en buena medida por una capacidad de agencia socialmente asignada, esto es, la de ser la cabeza de familia, que toma decisiones y provee al hogar. Caso contrario, en principio, es el de las enfermeras, cuya profesión es fundamentalmente femenina y en la que una parte de las profesionistas ocupan una posición subordinada en la estructura familiar que las convierte, al menos en una etapa de su vida, en seguidoras de sus parejas.

Con relación a la motivación o la causa de la migración, que es uno de los elementos que proponen algunos de los estudiosos de la migración, en los casos aquí tratados mayoritariamente se relacionan con varios motores (driving forces), como el deseo de superación o de aprendizaje, o con

\section{0}


motivaciones estrictamente económicas. Generalmente estos motores están interrelacionados y unos son más importantes que otros en distintas tipologías y en distintas etapas de la vida profesional. Predomina entre los ingenieros, en todo caso, una motivación proactiva más que reactiva ante una situación laboral insatisfactoria. Es importante destacar que algunos de estos ingenieros mencionan como causa de la migración el hecho de que el tipo de trabajo y las competencias que desarrollan en los Estados Unidos son raros en México debido a las diferencias en la estructura económica y en el nivel tecnológico de los dos países.

Las redes que se establecen a partir del primer trabajo juegan un papel fundamental en las dos profesiones. Estas redes, y las que se van construyendo posteriormente, son un canal muy importante para el acceso a empleos en los Estados Unidos. En el caso de los ingenieros, surgen a partir de dos vías: el ingreso desde el comienzo de la carrera laboral en una empresa trasnacional que sitúa al entrevistado en una trayectoria de empresa, o su ubicación en clusters industriales en algunas regiones de México.

Otro tipo de redes y de institucionalidad que también contribuye a la construcción de una carrera en los Estados Unidos es el de los estudios, ya sea de licenciatura, de maestría o de doctorado. Los grados obtenidos en los Estados Unidos son un certificado formal que otorga una fuerte legitimidad en el mercado de trabajo (Iredale, 2001).

Sin embargo, las redes, sobre todo para la búsqueda de empleo, pueden ser sustituidas por herramientas tecnológicas como los sitios web donde se ofrecen empleos.

En el caso de las enfermeras, las redes son conformadas por dos tipos de actores cuya presencia generalmente es excluyente en las trayectorias: los agentes subcontratistas y los familiares en Estados Unidos. Un aspecto relevante que ocasiona el contacto con una u otra red es un perfil diferencial entre las enfermeras. Típicamente, las enfermeras 
que emigraban a través de las empresas reclutadoras eran más jóvenes, con poca experiencia laboral, no tenían hijos y contaban con disponibilidad para viajar en cualquier momento, mientras que las que tenían trayectorias insertas en redes familiares frecuentemente manifestaron amplia experiencia laboral en México, estaban casadas y tenían dependientes económicos.

Con respecto a la estrategia de construcción de la trayectoria, se observa entre los ingenieros que en general estos no tienen un plan definido para trabajar de manera permanente en los Estados Unidos, sino que los planes van surgiendo a partir de las trayectorias en las empresas y en los mercados de trabajo. En varios casos, las estrategias se adecúan a la necesidad de conciliar estudios y carrera profesional o vida profesional y vida familiar, pues se observa que uno de los costos de la disponibilidad continua de los ingenieros hacia las empresas es la dificultad para hacer compatible una vida profesional exitosa y una vida familiar con atención a la pareja y a los hijos. En el caso de las enfermeras, se observaron dos vertientes: una en la que se da prioridad a la carrera profesional y otra en la que se le da a la reunificación familiar y el cuidado gratuito de enfermos consanguíneos.

El rol de género entre las enfermeras que migraron por causas familiares fue en algunos casos un factor inhibidor en la inserción laboral dentro del sector salud. El cuidado de tiempo completo de los hijos pequeños significó una tarea que justificaba para ellas alejarse de manera transitoria de su práctica profesional. Al mismo tiempo, sus restricciones financieras y temporales dejaban como única opción para ellas ocuparse en trabajos informales de medio tiempo no relacionados con sus estudios. De acuerdo con Kofman y Raghuram (2006), el nivel de calificación de las mujeres que migran como esposas de otros migrantes calificados generalmente no se toma en cuenta en el país destino, lo

\section{2}


que significa una negación de la importancia económica de la migración familiar o de la posible contribución de estas mujeres al mercado laboral. Estos autores señalan un desbalance de género expresado en una mayor dificultad para que las mujeres gocen de autonomía económica, incluso si su nivel educativo es similar o superior al de los hombres. Como consecuencia, las mujeres que entran a Estados Unidos por la vía familiar encuentran mayores barreras para cumplir sus objetivos laborales y profesionales.

También la regulación estatal tiene un papel importante en la legitimación de las competencias y los títulos mediante las facilidades que se les otorgan a los ingenieros para obtener un estatus legal. En este caso, facilitan este trámite el Estado del país de destino y, en alguna medida, la legislación que surge del TLCAN. Este tipo de regulaciones significa a su vez un apoyo fundamental para los intereses de las empresas trasnacionales y de los ingenieros, y no una restricción regulatoria.

En el caso de los ingenieros, uno de los actores fundamentales es la empresa y sus distintos roles, los cuales juegan en función de intereses, culturas organizativas, ciclos económicos y otros factores. En la trayectoria de empresa, la empresa es fundamentalmente protectora: tramita el estatus migratorio de los ingenieros, facilita los traslados de país o de ciudad y mantiene bajo su tutela a los profesionales. En el caso de las trayectorias de adaptación y riesgo, la empresa es parte de una trama de organizaciones que se encargan de organizar el proceso de outsourcing. En este caso, la pertenencia a la empresa es mucho más difusa y el estatus del ingeniero contratado por outsourcing es inestable y está sujeto a cambios, con un grado notable de incertidumbre. En la trayectoria estratégica, por otra parte, la legitimidad profesional del ingeniero migrante viene avalada en primera instancia por la obtención de un título de posgrado en el país de destino, Estados Unidos. 
En este caso, es el sistema educativo el que abre la vía para la obtención de un estatus legal seguro que puede llevar a la obtención de la ciudadanía. En la variante que se ha denominado pragmática, es de destacar que la concepción del ingeniero acerca de su trayectoria de mediano plazo lo hace privilegiar el aprendizaje sobre la seguridad. Sus credenciales están fundamentadas en una trayectoria en empresas de prestigio en México y en la apertura a nuevos aprendizajes. Finalmente, en el caso de las trayectorias precarias es en el que es más evidente la industria de la migración, pues en estas trayectorias hacen su aparición agentes con afán de lucro y prácticas fraudulentas.

Es decir, en conjunto, la regulación de las ingenierías resulta menos rígida a la entrada al mercado laboral de Estados Unidos que en el caso de las enfermeras. En función del tipo de trayectoria, las prácticas de los distintos actores ocupan un lugar central o accesorio: las empresas, el sistema educativo, los actores de la industria de la migración (empresas de outsourcing, reclutadoras virtuales, reclutadoras fraudulentas), todos influyen de manera diferente en función del tipo de trayectoria.

En el caso de las enfermeras, es claro que las certificaciones exigidas por las asociaciones de enfermería de Estados Unidos son una barrera que las enfermeras mexicanas deben salvar. En función de esta regulación de tipo estructural, se observan dos tipos de trayectorias. Las primeras son las trayectorias cuya motivación es profesional, en las que se reconocen dos secuencias importantes: una secuencia precaria, que precede a la obtención de la certificación, y una secuencia de despliegue y normalización posterior a ella. En la temporalidad de esta trayectoria, la precariedad es ocasionada en parte por las prácticas de los actores de la industria de la migración, como las agencias intermediarias que imponen altos costos a las enfermeras mexicanas. 
El segundo tipo de trayectoria es la de aquellas enfermeras cuya migración está muy influida por su condición de género y en las que, por tanto, su objetivo al migrar no es de tipo profesional. Sin embargo, después de una secuencia en la que no ejercen ningún tipo de trabajo, obtienen información suficiente para regularizar su residencia y pueden llegar a desarrollar una trayectoria laboral relativamente ascendente. En otros casos, sin embargo, se insertan en un segmento secundario, en el que se dedican a trabajos de cuidado con escasa estabilidad, bajos salarios y sin prestaciones laborales.

Abbott, A. (1988). The System of Professions. An Essay on the Bibliografía Division of Expert Labor. Estados Unidos:The University of Chicago Press.

Acacio, K. A. S. (20II). Getting Nurses Here: Migration Industry and the Business of Connecting Philippine-Educated Nurses with United States Employers (tesis de doctorado inédita). University of California: Berkeley.

Ariza, M., y Gandini, L. (20I2). "El análisis comparativo cualitativo como estrategia metodológica”, en M. Ariza, y L. Velasco (coords.), Métodos cualitativos y su aplicación empírica. Por los caminos de la investigación sobre migración internacional (PP. 497-537). México: IIS-UNAM/El Colef.

Arroyo de Cordero, G., y Jiménez Sánchez,J. (2005).“Repercusiones de la escasez versus migración de enfermeras como fenómeno global”. Revista de Enfermería IMSS, I3(I), 33-40.

Becker, H.S. (2009). “Préface”, en D. Didier,y C. Gadéa (eds.), Sociologies des groupes professionnels (pp. 9-13). París: La Découverte.

Benner, C. (2006). “El trabajo en la sociedad red:lecciones de Silicon Valley”, en M. Castells (ed.), La sociedad red: una visión global (pp. 226-253). Madrid:Alianza Editorial. 
Bibliografía
Brandi, C. (2006). "La historia del brain drain". Revista lberoamericana de Ciencia y Tecnología (CTS), 3(3), 65-85.

Bureau of Labor Statistics (20I2). "Occupational Outlook Handbook”. United States Department of Labor. Recuperado de: https://www.bls.gov/ooh

Calva, L. E., y Alarcón, R. (20I5)."La integración laboral precaria de los migrantes mexicanos calificados en Estados Unidos al inicio del siglo XXI". Papeles de población, 2 I (83), 9-39.

Carrión,V. (I8 de septiembre de 20I2). Entrevista personal a S. L., ingeniero aeronaútico.Tijuana, México.

(20I4). La migración de ingenieros mexicanos a Estados Unidos: un análisis de trayectorias profesionales (tesis de doctorado inédita). El Colegio de la Frontera Norte: Tijuana.

_ y Hualde, A. (20I3). “PProfesionales sin fronteras? Una aproximación a las trayectorias laborales de los ingenieros mexicanos en Estados Unidos”. Revista Latinoamericana de Estudios del Trabajo, I8(30), 7I-I02. Connell, J. (2008). “Towards a global health care system?", en J. Connell (ed.), The international migration of health workers (pp. I-29). Nueva York: Routledge.

Demazière, D., y Gadea, C. G. (2009). “Introduction”, en D. Demazière, y C. G. Gadea (eds.), Sociologies des groupes professionnels (pp. 13-27). París: La Découverte.

Didou, S. (2004). “Fuga de cerebros o diásporas? Inmigración y emigración de personal altamente calificado en México". Revista de la Educación Superior, 4(I32), 7-25.

Dubar, C., y Tripier, P. (1998). Sociologie des professions. París: Armand Colin.

Faulconbridge, J. R., y Muzio, D. (20I2). "Professions in a globalizing world:Towards a transnational sociology of the professions". International Sociology, 27(I), I 36-I52.

Freidson, E. (200I). "La teoría de las profesiones”. Perfiles educativos, 23(93), 28-43. 
Giddens, A. (2006). La constitución de la sociedad: bases para Bibliografía la teoría de la estructuración. Buenos Aires:Amorrortu. Hernández León, R. (20I2). “La industria de la migración en el sistema migratorio México-Estados Unidos". Trace. Travaux et recherches dans les Amériques du Centre, (6I), $4|-6|$.

Hualde, A. (2000). "La sociología de las profesiones en América Latina: iasignatura pendiente?”, en E. De la Garza, (coord.), Tratado latinoamericano de sociología del trabajo (pp. 664-678). México: Fondo de Cultura Económica.

Hugman, R. (199I). Power in caring professions. Hong Kong: MacMillan.

Iredale, R. (200I).“The Migration of Professionals:Theories and Typologies". International Migration, 39(5), 7-26. doi: I0.1 I I I/I468-2435.00169

Joel, L. A., y Kelly, L.Y. (2002). The nursing experience:Trends, challenges, and transitions. Estados Unidos: McGraw-Hill/ Appleton \& Lange.

Kofman, E.,y Raghuram, P. (2006)."Gender and global labour migrations: Incorporating skilled workers". Antipode, 38(2), 282-303.

Koser, K., y Salt, J. (1997). "The geography of highly skilled international migration". International Journal of Population Geography, 3(4), 285-303.

Lasserre, H. (1989). Le pouvoir de l'ingénieur.París: L'Harmattan. Lozano, F., y Gandini, L. (20I2). “La migración calificada de México a Estados Unidos: tendencias de la última década 2000-20 I0”. Coyuntura demográfica, (2), 5 I-55.

Gandini, L., y Jardón, A. E. (20I5). Condiciones laborales en tiempos de crisis: un análisis de la migración calificada de América Latina y el Caribe en Estados Unidos. Cuernavaca: CRIM/UNAM. 
Bibliografía

Nigenda, G., y González Robledo, L. M. (2005). Trade and health between Mexico and the U. S. Centre for Social and Economic Analysis. México: Mexican Health Foundation. y Ruiz, J. A. (coords.) (20I0). Formación, empleo y regulación de los recursos humanos para la salud. Bases para su planeación estratégica. Colombia: INSP/Cendex.

OIT (2010). Informe mundial sobre salarios 20 I0/20 I I:políticas salariales en tiempos de crisis. Santiago de Chile: OIT.

Pacheco,T.(1997)."La institucionalización del mundo profesional” en T. Pacheco, y A. Díaz Barriga (coords.), Cinco aproximaciones al estudio de las profesiones (pp. 27-39). México: CESU/UNAM.

Panaia, M. (2008). Una revisión de la sociología de las profesiones desde la teoría crítica del trabajo en la Argentina. Santiago de Chile: Naciones Unidas.

Portes, A., y Böröcz, J. ( 1989). “Contemporary immigration: Theoretical perspectives on its determinants and modes of incorporation". International Migration Review, 23(3), 606-630.

Rodríguez, A. (2004). Programa de certificación para los enfermeros mexicanos en Estados Unidos. Caso de estudio: programa de preparación de profesionistas en enfermería mexicanos (tesis de maestría inédita). Universidad de las Américas: Puebla.

Rodríguez Gómez, R. (20I3)."EI TLCAN y las profesiones: un estado de la cuestión". Revista de la Educación Superior, 42(167), 197-223.

Ros, J. (20I3).Algunas tesis equivocadas sobre el estancamiento económico de México. México: El Colegio de México/ Universidad Nacional Autónoma de México.

Rosales, Y. (05 de enero de 2013a). Entrevista personal a Susana. San Diego, California, Estados Unidos. (04 de marzo de 20 I 3b). Entrevista personal a Lucía. Los Ángeles, California, Estados Unidos.

\section{8}


(20I4). La inmigración de enfermeras mexicanas en

Bibliografía

California. Procesos de inserción laboral dentro y fuera del sector salud (tesis de doctorado inédita). El Colegio de la Frontera Norte:Tijuana.

Ruggles, S., Genadek, K., Goeken, R., Grover, J., y Sobek, M. (20I5). Integrated Public Use Microdata Series: Version 6.0 (American Community Survey). Minneapolis: University of Minnesota. doi: I0. I8I 28/D0 I0.V6.0

Sengenberger,W. (1988). “Dinámica de la segmentación del mercado de trabajo”, en W. Sengenberger (comp.), Lecturas sobre el mercado de trabajo en la República Federal de Alemania (pp. 345-377). Madrid: Ministerio de Trabajo y Seguridad Social.

Squires, A., y Beltrán, H. (20I3). Strengthening health systems in North and Central America: What role for migration? Washington: Migration Policy Institute.

Stark, O., y Fan, C.S. (2007).“'Losses and gains to developing countries from the migration of educated workers: An overview of recent research, and new reflections". CPPS Working Paper Series No. 183. Recuperado de: http:// commons.In.edu.hk/cppswp/87

Sullivan, S. E. (1999). "The changing nature of careers: A review and research agenda". Journal of Management, 25(3), 457-484.

White, H. C. (1958). The Organization Man. Nueva York: Simon and Schuster.

Zhou,Y.,y Hsu,J.Y.(20I I).“Divergent engagements: roles and strategies of Taiwanese and mainland Chinese returnee entrepreneurs in the IT industry". Global networks, I I (3), 398-419.

Zoya, R. L. (20I I). “El método comparado y la teoría de los sistemas complejos. Hacia la apertura de un diálogo interdisciplinario entre la ciencia política y las ciencias de la complejidad”. Reflexión Política, I3(25), 78-92. 